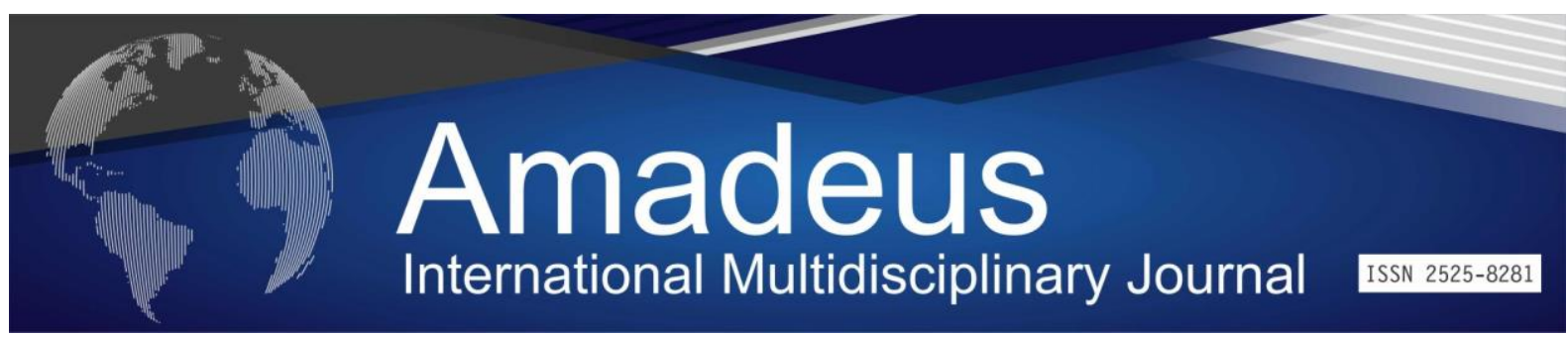

DOI: 10.14295/aimj.v4i8.110

\title{
Radiological Images of Pulmonary Thromboembolism in a Patient with the Diagnosis of Covid- 19: A Case Report
}

Larissa de Carvalho Bezerral,

Glauber Magalhães Pires ${ }^{2}$,

Helen Fernanda de Oliveira Sousa ${ }^{3}$,

Lucas Tavares Cruz de Albuquerque ${ }^{4}$, Maria Gabriela Gomes de Morais ${ }^{5}$

Raphael Xenofonte Morais Pinheiro 6
Abstract: Sars - Cov-2 infection has been associated with clinical manifestations not only immunological, but also coagulative. A series of serological markers can increase its concentration when there is the presence of Thromboembolism (PTE) in patients with COVID-19, such as D-dimer, cardiac markers including cerebral natriuretic peptide, creatine kinase and troponin $\mathrm{T}$. The immune factors that contribute to COVID-19 intravascular coagulopathy are: diffuse damage and alveolar inflammation; diffuse interstitial inflammation, extensive activation of pulmonary macrophages, dysregulation of innate pulmonary immune responses (eg, down regulation of angiotensin 2-converting enzyme receptor expression), adaptive immune responses to COVID-19, activation of innate immunity with advanced age and age-related coagulation cascade changes. This article shows a case report of a patient with COVID-19 and Pulmonary Thromboembolism, correlating the aspects observed in the patient's image and clinic with the current literature.

Keywords: Coronavirus infections, Pulmonary embolism, Tomography.

\footnotetext{
${ }^{1}$ Acadêmica de medicina da Faculdade de Medicina Estácio de Juazeiro do Norte (FMJ) - Ceará, Brasil

Contato: larissadecarvalhob@hotmail.com

2 Acadêmico de Medicina da Faculdade de Medicina Estácio de Juazeiro do Norte (FMJ), Ceará, Brasil; Docente da Universidade Federal Rural de Pernambuco, Serra Talhada, Pernambuco, Brasil

Contato: glaubermpires@yahoo.com

${ }^{3}$ Acadêmica de medicina da Faculdade de Medicina Estácio de Juazeiro do Norte (FMJ) - Ceará, Brasil

Contato: helennfernanda.hf@gmail.com

${ }^{4}$ Acadêmico de Medicina da Faculdade de Medicina Estácio de Juazeiro do Norte (FMJ), Ceará, Brasil

Contato: lucastavares47@gmail.com

${ }^{5}$ Acadêmica de medicina da Faculdade de Medicina Estácio de Juazeiro do Norte (FMJ) - Ceará, Brasil

Contato: gabimorais13@hotmail.com

${ }^{6}$ Radiologista docente da Faculdade de Medicina Estácio de Juazeiro do Norte (FMJ)- Ceará, Brasil

Contato: radioimip2011@gmail.com
} 


\title{
Imagens Radiológicas de Tromboembolismo Pulmonar em um Paciente com o Diagnóstico de Covid- 19: Um Relato de Caso
}

\begin{abstract}
Resumo: A infecção pelo Sars-Cov-2 tem se relacionado com manifestações clínicas não apenas imunológicas, mas também coagulativas. Uma série de marcadores sorológicos pode aumentar sua concentração quando há a presença de Tromboembolismo (TEP) em pacientes com COVID-19, tais como, o Ddímero, marcadores cardíacos incluindo o peptídeo natriurético cerebral, creatina quinase e troponina $\mathrm{T}$. Os Fatores imunes que contribuem para a coagulopatia intravascular da COVID-19 são: dano difuso e inflamação alveolar; inflamação intersticial difusa, ativação extensa de macrófagos pulmonares, desregulação das respostas imunes inatas pulmonares (como, por exemplo, regulação negativa da expressão do receptor da enzima conversora da angiotensina 2), respostas imunes adaptativas a COVID-19, ativação da imunidade inata com a idade avançada e alterações em cascata de coagulação relacionadas à idade. Esse artigo mostra um relato de caso de um paciente com COVID-19 e Tromboembolismo Pulmonar, correlacionando os aspectos observados na imagem e na clínica do paciente com a literatura vigente.
\end{abstract}

Palavras-chave: Coronavirus infections, Pulmonary embolism, Tomography.

\section{Introdução}

A infecção pelo Sars - Cov - 2 tem se relacionado com manifestações clínicas não apenas imunológicas, mas também coagulativas. Vários fatores desregulatórios imunes favorecem o surgimento de coagulopatias intravasculares, com agressão direta ao endotélio. O SARS-Cov2 possui tropismo para os pneumócitos tipo II, ocasionando inibição da expressão do receptor de enzima conversora de angiotensina 2, que associado ao quadro inflamatório alveolar e intersticial conduz a um processo hiper coagulativo, com síndrome de ativação macrofágica e de fatores de coagulação (McGonagle, S O’Donnell, Sharif, Emery \& Bridgewood, 2020).

Nessa perspectiva, é notório que pacientes com COVID-19 apresentam características imuno-angiogênicas caracterizadas pela existência de dano alveolar difuso com presença de células inflamatórias na microvasculatura e surgimento de trombos que podem ocasionar obstrução de vasos, gerando danos pulmonares, dentre eles infarto pulmonar e necrose hemorrágica acentuada (McGonagle et al, 2020). Além disso, com o desenvolvimento da doença há o surgimento de um processo de neovascularização através de um mecanismo de angiogênese principalmente intussusceptiva que se pressupõe favorecer os eventos 
trombóticos (Ackermann et al, 2020). Vale ressaltar ainda, que a ventilação positiva danifica as barreiras alveolares endoteliais interferindo e favorecendo também, de forma iatrogênica, os mecanismos imuno-angiogênicos da doença (McGonagle et al, 2020).

Desse modo, uma série de marcadores sorológicos podem aumentar de concentração quando há a presença de Tromboembolismo (TEP) em pacientes com COVID19 como o D-dímero, marcadores cardíacos incluindo o peptídeo natriurético cerebral, creatina quinase e troponina $\mathrm{T}$ (McGonagle et al, 2020). Além disso, muitas comorbidades aumentam a chance do surgimento de TEP em pacientes com COVID-19 tendo destaque sua manifestação em pacientes obesos (Poyiadi et al, 2020; Phend 2020).

Sendo assim, as manifestações radiológicas, principalmente na tomografia computadorizada (TC), são de extrema importância tanto na triagem e diagnóstico precoce quanto no acompanhamento de pacientes com COVID-19 a fim de detectar lesões pulmonares (Wang et al, 2020). Dessa forma, o objetivo desse artigo é mostrar um relato de caso de um paciente com COVID-19 e TEP correlacionando os aspectos observados na imagem e na clínica do paciente com a literatura vigente.

\section{Relato do Caso}

Paciente masculino, hígido, 33 anos, procurou atendimento médico devido a quadro febril e queixa de dispneia. Relata que está no sétimo dia de síndrome gripal e foi verificada saturação de $89 \%$. Posteriormente, foi encaminhado à Emergência de um Hospital de Juazeiro do Norte e ao ser avaliado clinicamente, exibe um exame físico que destaca um bom estado geral e avaliação do aparelho cardiovascular sem alterações, no entanto, na avaliação do aparelho respiratório, percebeu-se presença de estertores crepitantes difusos em terço superior do pulmão esquerdo. Devido ao quadro respiratório, foi realizada radiografia do tórax (Figura 1) evidenciando consolidação em lobo superior pulmonar à esquerda. Realizou também D-dímero com resultado de $5.000 \mathrm{ng} / \mathrm{dl}$. 
Figura 1: Radiografia de tórax em PA evidenciando opacidade consolidativa em campo pulmonar superior à esquerda de provável etiologia inflamatória/infecciosa de acordo com a apresentação clínica do paciente.

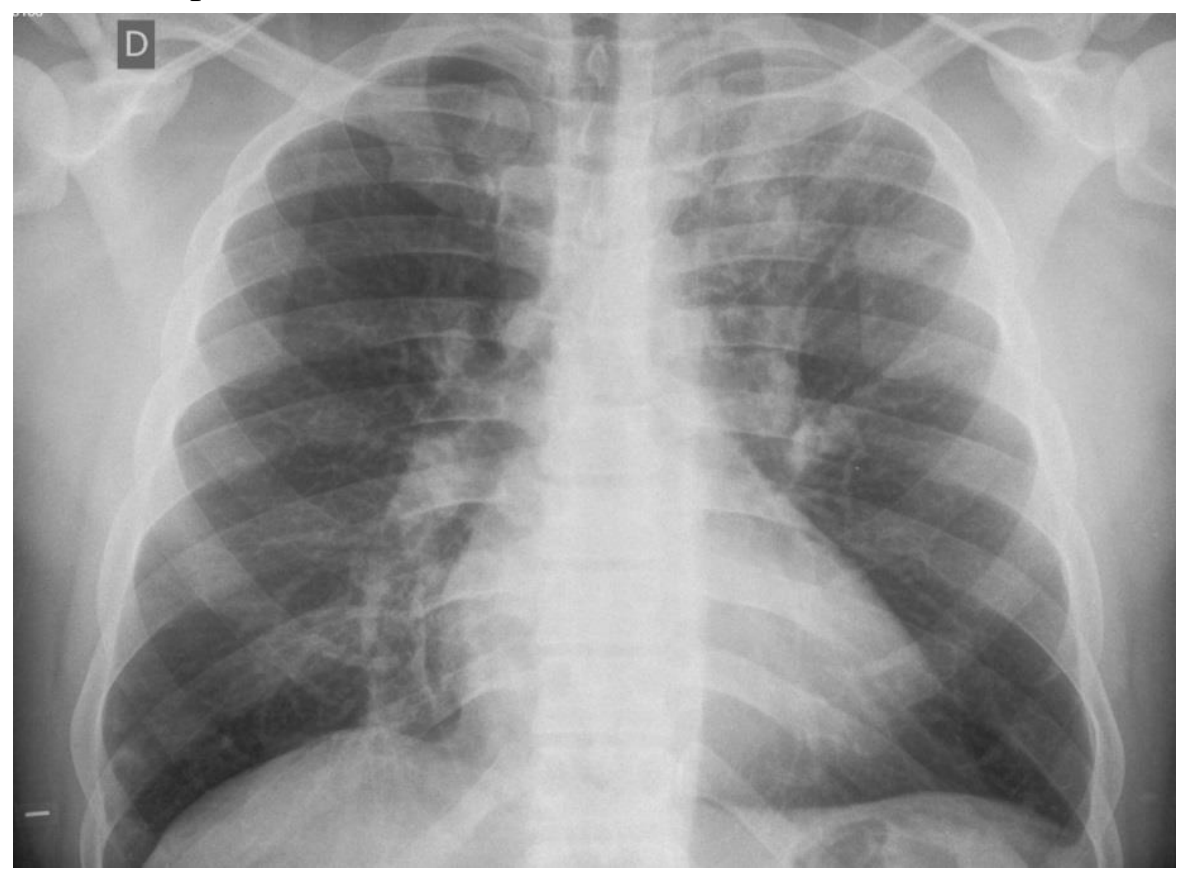

Fonte: Imagens do estudo.

No $10^{\circ}$ dia de internação, dando seguimento a investigação de Covid-19, apesar do padrão radiográfico não típico de infecção pelo Sars-Cov-2 e sugestivo de pneumonia lobar, foi realizado PCR-RT que resultou positivo para coronavírus. Em seguida, o paciente evolui com piora da dispneia associada à queda da saturação, porém a pressão arterial se manteve normal, a frequência cardíaca estava em valores normais e o D-dímero se alterou para 70.000 ng/dl. Tendo em vista essas alterações, foi realizada uma TC de tórax (Figuras 2 e 3) e iniciado protocolo para Tromboembolismo Pulmonar e eco-color doppler venoso dos membros inferiores a fim de pesquisar trombose venosa profunda (TVP) e esse exame demonstrou ausência de sinais sugestivos de TVP em membros inferiores enquanto que na angiotomografia computadorizada de tórax (Figura 4), foi constatada presença de trombos bilaterais segmentares pulmonares com alteração de perfusão. Dessa forma, estando estabelecido o diagnóstico de Tromboembolismo Pulmonar, fez-se o tratamento com droga antitrombótica e após melhora do quadro, o paciente teve alta/cura. 
Figura 2: Tomografia computadorizada de tórax, janela pulmonar, corte axial, mostrando opacidades em vidro fosco de aspecto central e periférico, achados de padrão indeterminado para Covid-19. As opacidades periféricas podem estar associadas a fenômenos tromboembólicos.

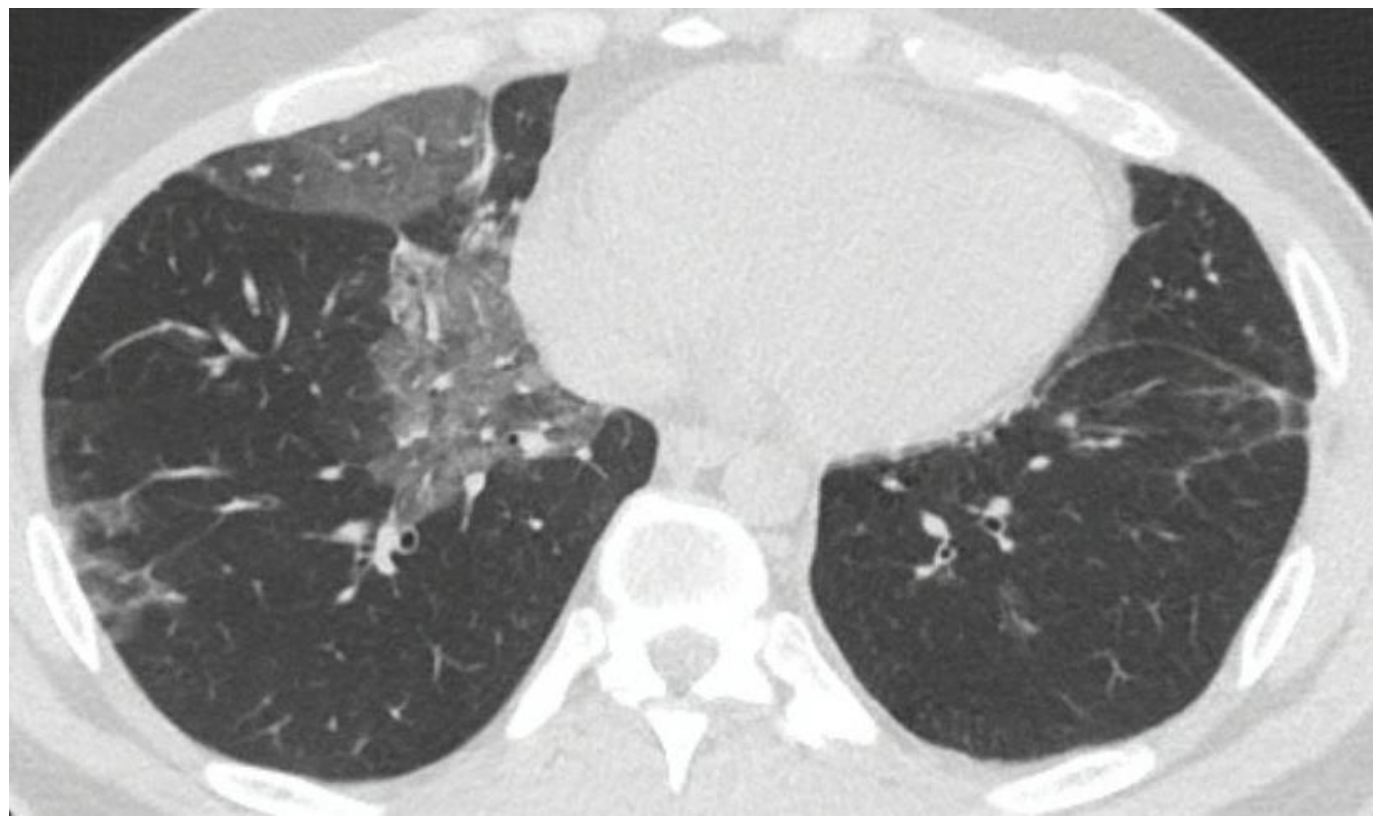

Fonte: Imagens do estudo.

Figura 3: Tomografia computadorizada do tórax, janela pulmonar, corte axial, evidenciando consolidação associada à presença de opacidade em vidro fosco em segmento ápico-posterior do pulmão esqerdo, provável etiologia inflamatória/infecciosa, vista na radiografia de tórax (Figura 1).

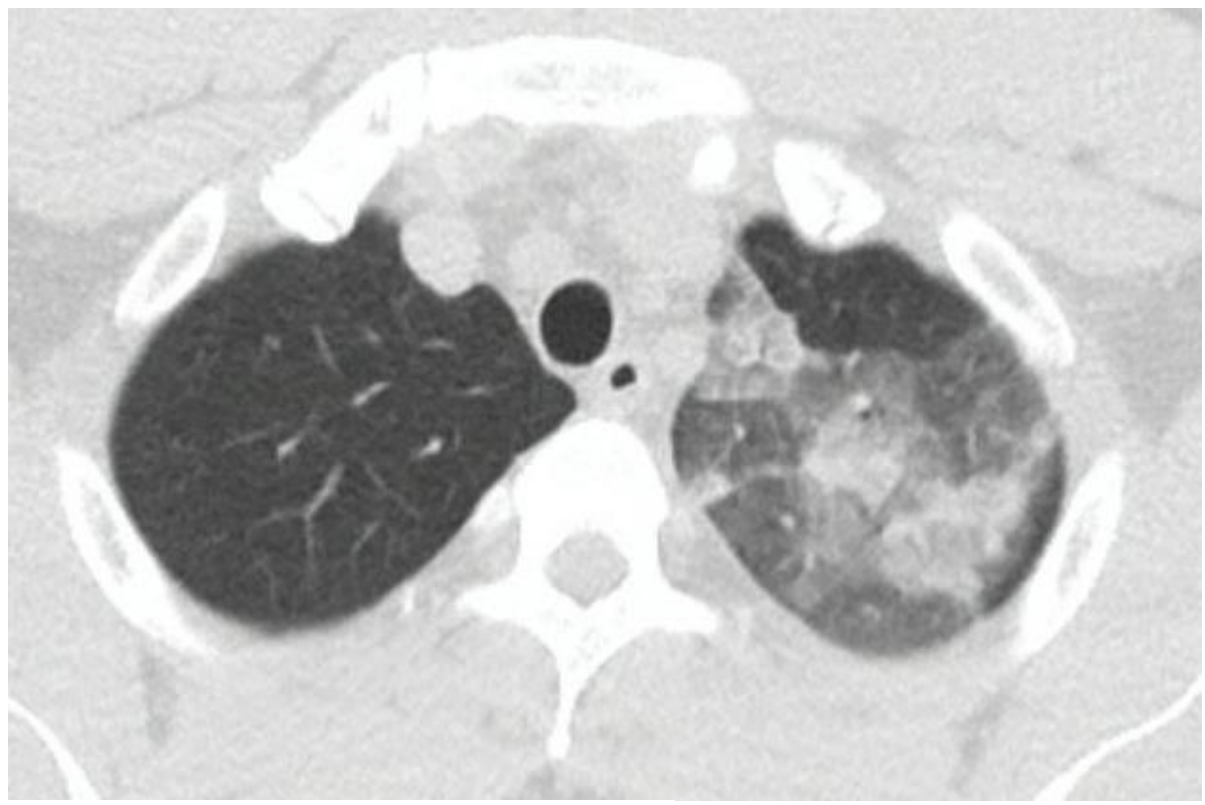

Fonte: Imagens do estudo. 
Figura 4: Imagens de angiotomografia do paciente mostrando presença de falha de enchimento em artérias segmentares pulmonares bilateralmente, compatíveis com trombose arterial pulmonar em (A) e (B).

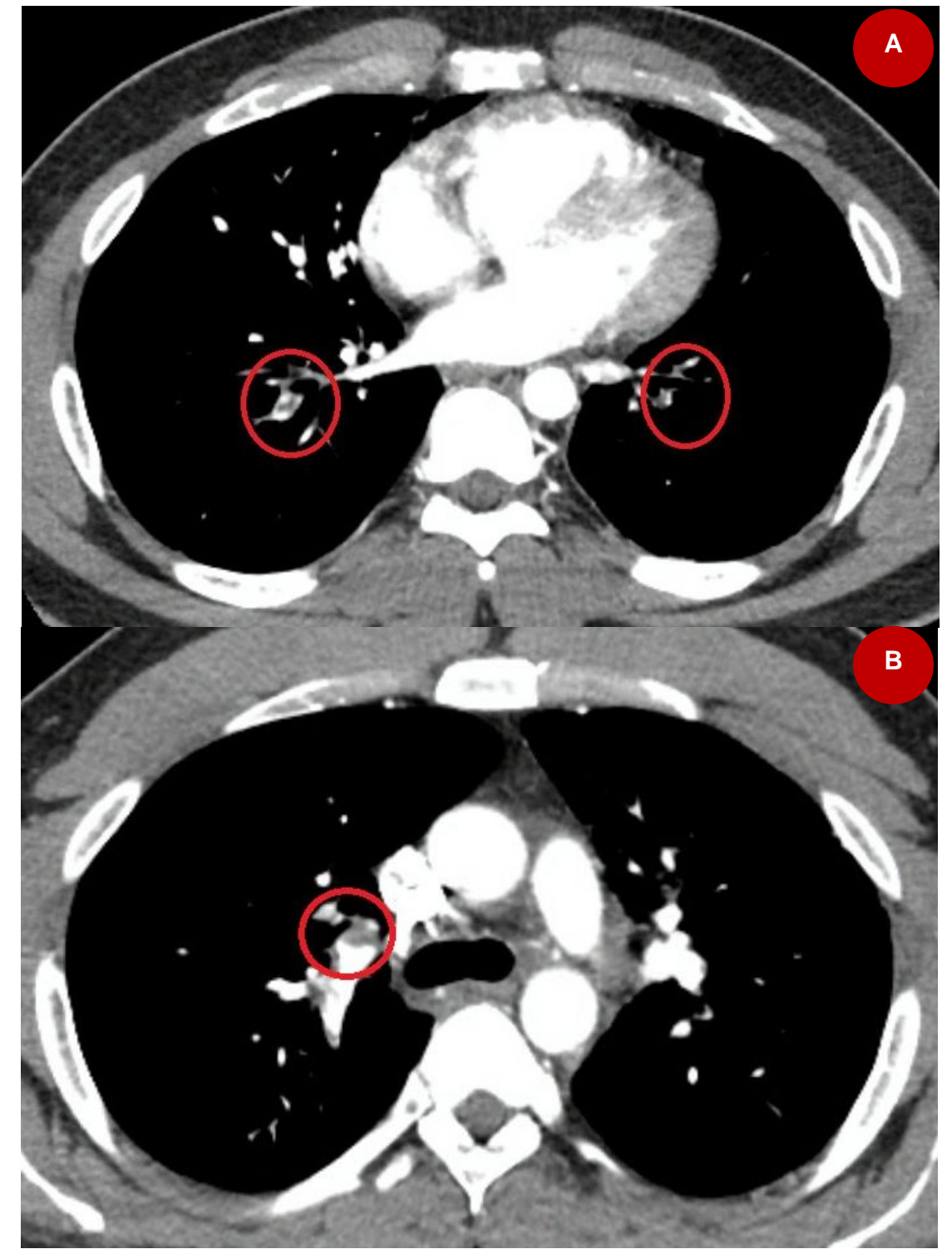

Fonte: Imagens do Estudo

\section{Discussão}

Nesse caso, observou-se inicialmente que a radiografia do paciente mostrou um padrão radiográfico não típico e sugestivo de pneumonia lobar. Na literatura, o valor diagnóstico da radiografia de tórax é relativamente baixo em 30 a $60 \%$ na pneumonia por COVID-19. Embora seja possível observar algumas anormalidades na pneumonia viral nas radiografias de 
tórax, não pode ser excluída a doença se a radiografia de tórax for normal ou não sugestiva, como a desse relato de caso (Akçai, Ozlu \& Yilmaz, 2020). Por isso, é necessário uma tomografia computadorizada precoce, já que o exame demonstra características de curto tempo e resolução de alta densidade. O valor diagnóstico da TC de tórax reside principalmente na detecção de lesões (mesmo lesões precoces que são não são visualizadas na radiografia), caracterização das lesões e avaliação da gravidade para facilitar a classificação e o tratamento clínico (Wang et al, 2020). A Sociedade Radiológica da América do Norte (RSNA), Sociedade de Radiologia Torácica (STR) e a American College of Radiology (ACR) classificaram os achados de Covid-19 dna TC de tórax em grupos:

- Típico: Características típicas de imagem relatadas geralmente maiores para especificidade para pneumonia por COVID-19, aspecto periférico, bilateral (multilobar), consolidação ou linhas intralobulares visíveis (aspecto de pavimentação em mosaico), padrão de lesão com halo invertidos e pneumonia em organização.

- Indeterminado: Recursos de imagem inespecíficos/indeterminados da pneumonia por COVID-19, aspecto focal ou multifocal, peri-hilar, unilateral ou não-arredondado ou não periférico podem ser vistos com a pneumonia por COVID-19.

- Atípico: Características incomuns ou não relatadas da pneumonia por COVID-19, onde há consolidação lobar ou segmentar isolada, pequenos nódulos discretos, cavitação ou espessamento septal interlobular, derrame pleural. Os recursos de imagem são atípicos ou raramente relatados para pneumonia por COVID-19. Diagnóstico alternativo deve ser considerado.

- Negativo: Não há características da pneumonia, nem características para sugerir pneumonia na TC de tórax (Akçai, Ozlu \& Yilmaz, 2020).

A TC do nosso paciente mostrou opacidades em vidro fosco de aspecto central, perihilar, pouco periférico de aspecto indeterminado para Covid-19.

Devemos destacar que no relato de alguns estudos relacionados ao Tromboembolismo Pulmonar em pacientes diagnosticados com COVID- 19, a embolia pulmonar aguda era comum em casos de COVID-19, geralmente em pacientes obesos e naqueles que não estavam doentes o suficiente para exigir cuidados intensivos (Phend, 2020). O paciente relatado não apresentava comorbidades e não exigiu cuidados de terapia intensiva.

Inicialmente, nosso paciente apresentou um D-dímero de $5.000 \mathrm{ng} / \mathrm{dl}$, valores elevados desse marcador indicam a presença de níveis anormais de fibrina no organismo. Esses valores elevados podem significar uma TVP, TEP, mas também cirurgia, trauma ou infecção recente. 
Por isso, um D-dímero elevado associado à clínica de estertores crepitantes difusos em terço superior do pulmão esquerdo, possibilitaram a suspeita de algum desses diagnósticos, porém, o paciente se apresentava estável, saturação normal e radiografia de tórax atípica para COVID-19, então, não se prosseguiu a investigação para TEP. O seguimento do diagnóstico aconteceu no $10^{\circ}$ dia de internação, após resultado de PCR-RT ser positivo para a infecção por Coronavírus e evolução do quadro de dispneia associada à queda da saturação. Foi solicitado, então, um novo D-dímero, tendo o resultado de $70.000 \mathrm{ng} / \mathrm{dl}$, realizou-se, assim, uma TC do tórax mostrando aspecto indeterminado para o COVID-19.

Iniciou-se o protocolo para Tromboembolismo Pulmonar após descartar TVP pelo eco-color Doppler venoso dos membros inferiores e presença de falha de enchimento em artérias segmentares pulmonares bilateralmente, compatíveis com trombose arterial pulmonar na angio-TC. Dessa forma, o diagnóstico da TEP foi tardio e o D-dímero foi um marcador importante nesse processo.

É essencial o diagnóstico precoce da TEP associado ao COVID-19 e do D-dímero como marcador, pois, no estudo de Phend (2020), a autópsia das 12 primeiras mortes por COVID-19 em uma área da Alemanha resultou em trombose venosa profunda insuspeita em sete $(58 \%)$ e TEP como causa direta de morte em quatro e em uma série austríaca de 11 pacientes falecidos com COVID-19 (10 dos quais foram selecionados aleatoriamente para autópsia) mostrou que, embora não houvesse suspeita clínica de TEP antes da morte em nenhum dos pacientes, todos apresentavam trombose de pequenas e médias artérias pulmonares.

Já o estudo de Poyiadji et al (2020) usou D-dímero elevado e obesidade como preditores de TEP e encontrou que a terapia com estatinas antes da admissão foi associada a uma probabilidade $60 \%$ menor de TEP. Este estudo avaliou as características clínicas dos pacientes com COVID- 19 que desenvolveram TEP ao comparar seus marcadores inflamatórios, D-dímeros e seus desfechos, mostrou que 72 das 328 pacientes foram positivos para TEP (22\%) e D-dímero aumentado no grupo com EP. Só 51\% dos diagnósticos de TEP foram detectados na sala de emergência, $23 \%$ dos pacientes em profilaxia desenvolveram TEP e concluiu que o D-Dímero foi um bom preditor de TEP nesses pacientes.

A pneumonia por COVID-19 inclui um D-dímero elevado no plasma e concentrações em conjunto com marcadores cardíacos elevados, incluindo peptídeo natriurético cerebral, creatina quinase, e troponina T. A COVID-19 normalmente não desenvolve coagulação intravascular disseminada sistêmica aberta. Em casos raros de COVID-19 em que a 
coagulação intravascular disseminada se desenvolve, tende a restringir-se a doenças em estágio avançado, que não foi o caso do nosso paciente. Esse achado se reflete na observação consistente de que contagem de plaquetas e concentração de fibrinogênio não são substancialmente reduzidas em pacientes com COVID-19, apesar do aumento acentuado nas concentrações do D-dímero. O Fibrinogênio geralmente permanece elevado nesses pacientes, com uma resposta de fase aguda em andamento. A pneumonia por COVID-19 desencadeará a expressão de fator tecidual, tanto nas células endoteliais quanto nas células ativadas, infiltrando macrófagos e neutrófilos. O efeito líquido será a apresentação local do fator tecidual transmitido pelo sangue dentro dos pulmões, o que aumentará ainda mais a ativação da cascata de coagulação (McGonagle, O’Donnell, Sharif, Emery \& Bridgewood 2020).

Nosso caso relata a importância da angiotomografia computadorizada de tórax, já que foi constatada a presença de trombos bilaterais segmentares pulmonares bilaterais com alteração de perfusão, essenciais no diagnóstico. Dessa forma, com o diagnóstico de Tromboembolismo Pulmonar, fez-se o tratamento com droga antitrombótica, eficaz para a alta do paciente.

Para entender o motivo de alguns pacientes diagnosticados com COVID-19 apresentarem em sua evolução fenômenos tromboembólicos, como no relato de caso, o estudo de Ackermann et al (2020) demonstrou a fisiopatologia, histologia, morfologia e alterações moleculares em autópsias de pulmões de pacientes que faleceram por Covid-19, por vírus influenza A (subtipo H1N1) e pulmões de controle não infectados, obtendo como seus resultados que trombos de fibrina nos capilares alveolares puderam ser vistos em ambos os pulmões dos grupos de pacientes. Os micro-trombos capilares alveolares foram 9 vezes mais prevalentes em pacientes com Covid-19 do que em pacientes com influenza. Os membros da família Coronavírus mostram tropismo para a enzima conversora de angiotensina 2 em pneumócitos tipo II. Esse tropismo, juntamente com a estreita justaposição dos pneumócitos tipo II e o quadro inflamatório multifacetado grave na vascularização pulmonar são prováveis que conduza ao quadro pulmonar hipercoagulável generalizado observado em pacientes com COVID-19. Os Fatores imunes que contribuem para a coagulopatia intravascular de COVID19 são dano difuso e inflamação alveolar; inflamação intersticial difusa, ativação extensa de macrófagos pulmonares, desregulação das respostas imunes inatas pulmonares (por exemplo, regulação negativa da expressão do receptor da enzima conversora da angiotensina 2), respostas imunes adaptativas a COVID-19, ativação da imunidade inata com a idade 
avançada e alterações em cascata de coagulação relacionadas à idade (McGonagle, O’Donnell, Sharif, Emery \& Bridgewood, 2020).

\section{Conclusões}

O caso relatado e os trabalhos citados mostram a importância de investigar a COVID19 associado ao tromboembolismo pulmonar em pacientes que apresentam D-dímero elevado com clínica sugestiva de infecção pelo Coronavírus, já que esses pacientes apresentam fatores imunes que contribuem para uma coagulopatia intravascular, provocando um quadro hipercoagulável generalizado. Para essa investigação, os exames de imagem, como a angiotomografia de tórax, tomografia computadorizada de tórax, radiografia de tórax e ecocolor Doppler venoso de membros inferiores são importantes para elucidar o quadro. No caso apresentado, o TEP só foi diagnosticado no $10^{\circ}$ dia após a piora da dispneia, um novo exame com nível de D-dímero de 70.000 ng/dL e a realização de uma angiotomografia de tórax. Dessa forma, o uso desses preditores pode ajudar a evitar que casos passem despercebidos e facilitam o diagnóstico precoce e um melhor desfecho para os pacientes.

\section{Referências}

Ackermann, M., Verleden, S. E., Kuehnel, M., Haverich, A., Welte, T., Laenger, F., Vanstapel, A., Werlein, C., Stark, H., Tzankov, A., Li, W. W., Li, V. W., Mentzer, S. J., \& Jonigk, D. (2020). Pulmonary Vascular Endothelialitis, Thrombosis, and Angiogenesis in Covid-19. New England Journal of Medicine. DOI: https://doi.org/10.1056/nejmoa2015432

Akçai, S., Ozlu, T., Yilmaz, A. (2020). Radiological approaches to COVID-19 pneumonia. Turkish Journal of Medical Sciences, 50(3), 604-610. DOI: 10.3906/sag-2004-160

Gao, L., \& Zhang, J. (2020). Pulmonary High-Resolution Computed Tomography (HRCT) Findings of Patients with Early-Stage Coronavirus Disease 2019 (COVID-19) in Hangzhou, China. Medical Science Monitor: International Medical Journal of Experimental and Clinical Research, 26, e923885-1

Jiang, X., Yin, Z., Wang, T., Zhai, N., Lu, F., Zhan, C., Han, Q. e Feng, C. (2020). COVID19 Desempenho da Tomografia Computadorizada Dinâmica (CT) e Observação de Alguns Indicadores Laboratoriais. Medical science monitor: revista médica internacional de pesquisas experimentais e clínicas, 26, e924403. DOI: 10.12659 / MSM.924403. 
McGonagle, D., O’Donnell, J. S., Sharif, K., Emery, P., Bridgewood, C. (2020). Immune mechanisms of pulmonary intravascular coagulopathy in COVID-19 pneumonia. Lancet Rheumatol, 1-9. DOI: 10.1016/S2665-9913(20)30121-1

Phend, C. (2020). COVID-19 Pulmonary Embolism Often Seen Outside the ICU. Medpage Today. Acesso em 25 de maio de 2020 de https://www.medpagetoday.com/infectiousdisease/covid19/86535.

Poyiadji, N., Cormier, P., Patel, P. Y., Hadied, M. O., Bhargava, P., Khanna, K., Nadig, J., Keimig, T., Spizarny, D., Reeser, N., Klochko, C., Peterson, L., \& Song, T. (2020). Acute Pulmonary Embolism and COVID-19. Radiology, 201955.

Wang, K., Kang, S., Tian, R., Zhang, X., Zhang, X., \& Wang, Y. (2020). Imaging manifestations and diagnostic value of chest CT of coronavirus disease 2019 (COVID-19) in the Xiaogan area. Clinical radiology, 75(5), 341-347. DOI: 10.1016/j.crad.2020.03.004

Yoon, S. H., Lee, K. H., Kim, J. Y., Lee, Y. K., Ko, H., Kim, K. H., Park, C. M., \& Kim, Y.H. (2020). Chest Radiographic and CT Findings of the 2019 Novel Coronavirus Disease (COVID-19): Analysis of Nine Patients Treated in Korea. Korean Journal of Radiology, 21(4), 494. DOI: https://doi.org/10.3348/kjr.2020.0132

Yuan, M., Yin, W., Tao, Z., Tan, W. e Hu, Y. (2020). Associação de achados radiológicos com mortalidade de pacientes infectados com o novo coronavírus de 2019 em Wuhan, China. PloS one , 15 (3), e0230548. DOI: 10.1371 / journal.pone.0230548.

\section{How to cite this article (APA format):}

Bezerra, Larissa de Carvalho; Pires, Glauber Magalhães; Sousa, Helen Fernanda de Oliveira; Albuquerque, Lucas Tavares Cruz de; Morais, Maria Gabriela Gomes de; Pinheiro, Raphael Xenofonte Morais (2020). Radiological Images of Pulmonary Thromboembolism in a Patient with the Diagnosis of Covid- 19: A Case Report. Am. In. Mult. J., Feb to Jun. (8) 5, 98-101.

Received: 06/05/2020;

Accepted: 06/08/2020. 